nevertheless, the author is to be congratulated in having done what many biological authors have neglected to do.

Another fresh note is the chapter on the methods of science, which, if read by the discriminating student before and after reading the rest of the book, will prove invaluable in giving the reader the most desirable outlook on the subject. Apart from this common-sense, and therefore correct, method of treatment, it may be said that the book has an attractive style and is comparatively well illustrated. It can be whole-heartedly recommended to students up to matriculation standard.

(2) "The World of Nature" makes a different appeal : but its standard is equally high. It is more a natural history than a biology, and is written to make the world of Nature "intelligible to the ordinary educated person". We venture to say that it will not only do this, but it will also prove to the sceptical and disinterested what a fascinating field for cultural interest lies around them. There is only one criticism -a selfish one perhaps-that is, we would wish to see more of the unique but brilliantly executed illustrations.

\section{The Botanist in Ireland}

By Dr. Robert Lloyd Praeger. Pp. xii $+497+44$ plates. (Dublin : Hodges, Figgis and Co., 1934.) 12s. $6 d$.

THIs volume is difficult to classify. It is not a Flora ; it is not a guide book ; it is not a scientific treatise on Irish phytogeography; yet it combines features of all these into a homogeneous whole and provides those interested in the Irish flora with an ideal guide and companion for their studies and their travels. No one but Dr. Praeger could have written such a work, and it is indeed a fitting crown to his lifelong labours on the flora of his native land.

The work is divided into four parts. The first comprises sections on the physiography, geology, topography and climate of Ireland, each a model of compression and clear statement. The second contains notes on various miscellaneous aspects of the flora, including topographical and ecological distribution, and an invaluable list of rare or interesting Irish plants, in which information hitherto scattered in periodicals has been brought together.

The main part of the book is devoted to a topographical survey of the whole country, with a full description of the flora of each area. Dr. Praeger's intimate knowledge of every square yard that he describes gives to these pages a remarkable vividness which must be unique in botanical literature.

The concluding section consists of a census list of the flora, with the distribution of each species indicated by numbers referring to the counties in which it occurs. There is an excellent index.

The whole volume is copiously illustrated with maps, diagrams and photographs of individual plants and botanical localities. The photographs, most of which were taken by $\mathrm{Mr}$. R. Welch, are extraordinarily beautiful, and add greatly to the value of the book.

\section{Elementary Microtechnique}

By H. Alan Peacock. Pp. vii +200 . (London : Edward Arnold and Co., 1935.) 5s. 6d. net.

THIs small book should prove of value to the amateur and elementary biologist, and the more advanced student and laboratory worker may well add it to his reference library, for it contains a number of practical hints and useful dodges and recipes. The matter presented is concisely given, largely in tabular form. After some introductory remarks on protoplasm and eytology, the processes and principles of microtechnique and methods for making microscopical preparations are considered. A chapter follows on technique, including fixing, hardening, embedding and sectioning, staining, and mounting, with a section on bulk staining. The next chapter deals in alphabetical order with methods for specific purposes for animal and plant organisms and tissues, and contains a surprising number of headings, ranging from acarine disease of bees and annelids to Vorticella and yeast (rotifers do not seem to be mentioned here). The next chapter gives the use of stains, followed by one containing the formulæ.

Ink for writing on glass is not given, while grease pencils are so easily obtained that the method for making them might well be omitted. Similarly, such agents as Farrant and glycerin jelly are probably better purchased than made, and the same applies to eyepiece scales and micrometers. The remarks on numerical aperture may give rise to confusion, as the term "definition" is used, instead of the correct one, "resolution". A most useful appendix gives the sources from which the organisms and tissues mentioned may be obtained, and their preservation is dealt with in another appendix.

\section{R. T. H.}

\section{Chemistry}

Organic Syntheses:

an Annual Publication of Satisfactory Methods for the Preparation of Organic Chemicals. Carl R. Noller, Editor-in-Chief. Vol. 15. Pp. v+104. (New York : John Wiley and Sons, Inc.; London : Chapman and Hall, Ltd., 1935.) 8s. 6d. net.

ThIRTY substances are included in the current volume of this welcome annual. Among acyclic compounds with long carbon chains are $n$-dodecyl bromide (made from $n$-dodecyl alcohol), $n$-hexadecane (from cetyl alcohol, via cetyl iodide), and oleyl alcohol (from butyl oleate, prepared from olive oil by alcoholysis). Simple open-chain compounds include trichloroethyl alcohol, which is readily prepared by treating chloral with aluminium ethoxide in presence of anhydrous alcohol. In the aromatic series, a simple method is given for converting 2,4-dinitrochlorobenzene into 2,4-dinitroaniline by treatment with ammonium acetate and ammonia gas at $170^{\circ}$ in an open vessel ; further, an example of oxidation with selenium dioxide is provided by the preparation of phenylglyoxal from acetophenone. In the heterocyclic series, Hans Fischer submits details of the preparation of 2,4-dimethyl-3,5-dicarbethoxypyrrole and its conversion into 2,4-dimethylpyrrole, 